\title{
Mathematical Model of Coextrusion Molding of Polymer-concrete Building Structures
}

\author{
Valery Dyadichev ${ }^{l}$, Andrey Kolesnikov ${ }^{2}$, Aleksandr Dyadichev $^{l}$, Ekaterina Dyadicheva $^{l}$, \\ Sergey Menyuk ${ }^{l}$ and Svitlana Chornobay ${ }^{l}$ \\ ${ }^{1}$ V.I. Vernadsky Crimean Federal University, Pavlenko Str., 3, Simferopol, Crimea, 295000, Russia \\ ${ }^{2}$ Lugansk National University named after Vladimir Dal, Department of Automation and Computer- \\ Integrated Technologies, Molodezhnyi Sq., 20-A, Lugansk, 91034, Ukraine
}

\begin{abstract}
The research identifies the optimality criterion of the technological process of the coextrusion molding of polymer-concrete building structures in the form of volumetric capacity as the correlation between forms quantity and technological cycle time. The set of parameters value constraints is made. The set consists of kinematic constraints, extruder's maximum power constrain, maximum motor torque constraint for the extruder trouble-free operation, constraint on the stress rate of the shift between layers, constraint on product cooling time, and minimum melt viscosity constraint. As a result, the task of coextrusion molding process improvement reduces to the task of linear programming. With the help of mathematical modelling the dynamics of cooling process of the preform in the mould is studied. The paper scrutinizes the influence of bulging pressure on the plybond strength in the multilayer polymer system in the manufacturing process of different capacity items.
\end{abstract}

\section{Introduction}

The technology of the coextrusion processing of primary and second polymer materials and concrete mixes is quite a complex process. While studying the process it is necessary to take into consideration a significant amount of technological factors and phenomena which influence process-dependent parameters and design factors of the process [1-3].

Therefore, a mathematical model of coextrusion molding process is necessary for making coextrusion machines on the basis of this technology and identifying optimum process-dependent parameters of their functioning.

A modern specialist, working a machinery complex which performs the coextrusion processing of polymer and concrete mixes, has to master the methodology and algorithms of doing these optimization tasks of choosing the optimum process condition. This becomes especially topical while operating expensive programmatically controlled plant [4-5], but setting the optimum process condition is implemented on the basis of knowing the physics process of coextrusion of polymers and concrete mixes, task mathematical modelling, application of computational algorithms search for the solution.

\footnotetext{
* Corresponding author: mr.dyadichev@ mail.ru
} 
During the coextrusion processing there always appear tasks of choosing the most effective solution variant out of the range of technically (technologically) acceptable ones [6-7]. While planning the technological process of manufacturing the product, these tasks acquire particular forms connected with the choosing of process-dependent parameters for a definite machine, materials, and a product being manufactured.

\section{Methods}

The developing mathematical model allows classifying the initial optimization task as the mathematical programming task which, depending on the limit type and the target function, can be done by using the methods of linear programming.

\section{Results}

Any technological process must be effective (optimum), i.e. the best one from the position of the chosen optimality criterion $\mathrm{K}_{\mathrm{o}}$. At the same time, various limit type requirements, which form the region of feasibility, must be met. For a definite technological process variant of the coextrusion processing of polymers and concrete mixes such a thesis means the necessity of calculating the optimum condition of the coextrusion processing, i.e. the search for optimum value of metrics $\mathrm{X}$ of the technological process.

Apart from the metrics of the technological process, there is a range of phase parameters $\mathrm{Y}$ which are the function of the metrics (motor's instantaneous output torque, drive power, shear stress in a polymer-concrete preform and others). Besides, the mathematical model of the process includes the metrics $\mathrm{V}$ - coefficients of equations and other constants for the current task [8].

All the limitations are presented as a system of inequalities, each of them gives the acceptance limits of the variation of the relevant phase or the required parameter (for example, the maximum of auger rotation frequency, the overload capacity of shear stress, etc). Analytically it can be put down as follows:

$$
\mathrm{X}_{\mathrm{i} \text { min }} \leq \mathrm{X}_{\mathrm{i}} \leq \mathrm{X}_{\mathrm{i} \text { max }}, \mathrm{Y}_{\mathrm{j} \text { min }} \leq \mathrm{Y}_{\mathrm{j}} \leq \mathrm{Y}_{\mathrm{j} \max } \text {. }
$$

Optimality criterion is the function of the initial and required parameters:

$$
\mathrm{K}_{\mathrm{o}}=\mathrm{K}_{\mathrm{o}}(\mathrm{X}, \mathrm{Y})
$$

They suppose, that this criterion has to be either maximized, or minimimized. The search for the vector of the required parameters, bringing the maximum (minimum) size of test $\mathrm{K}_{\mathrm{o}}$, is done on some range of feasible solutions.

The main purpose of the coextrusion machine is the processing of primary, second polymer raw materials and concrete mix for getting new high quality products with a high production process.

For the coextrusion molding process the following optimality criterion is proposed:

where $\mathrm{Q}$ - the volumetric capacity;

$$
K_{o}=Q=\frac{p}{\tau} \Rightarrow \max
$$

$\mathrm{p}$ - the quantity of forms;

$\tau-$ the cycle time of machine working.

The process is optimal when the optimum criterion Ko tends to the maximum in the sphere of process-dependent parameters limitation. 
The scrutiny of the optimum criterion shows that the criterion does not have extrema, i.e. it is monotonous. Thus, to find the optimal factors of the coextrusion molding it is necessary to research the size of test on the edge of the limit region.

During the research we exposed the following limits on the technological parameters value:

A. Kinematic constraints of minimum $n_{\min }$ and maximum $n_{\max }$ rotation frequency of the extruder drive $(\mathrm{N})$, correspondingly, as the following inequalities:

$$
N \geq n_{\text {min }}, N \leq n_{\text {max }} .
$$

Transformation of constrains (4) into efficiency constrains brings to the inequalities of the following type:

$$
Q \geq z_{n} n_{\min }, Q \leq z_{n} n_{\max },
$$

where $z_{n}$ - the coefficient depending on the geometrical shape of the extruder and the head:

$$
z_{n}=\frac{\alpha \cdot K_{\varepsilon}}{K_{\varepsilon}+\beta},
$$

where $\alpha$ - the coefficient of the forward flow of the extruder,

$\beta$ - the coefficient of the reverse flow of the extruder.

B. Constraint according to the maximum extruder power:

$$
Z_{p} \leq Z_{\text {eng }} \cdot C E_{e}
$$

where $Z_{\mathrm{p}}$ - power needed in the process of extrusion,

$Z_{\text {eng }}$ - engine power,

$\mathrm{CE}_{\mathrm{e}}$ - the extruder drive coefficient of efficiency.

The power needed in the process of coextrusion of multilayer polymers is identified according to the empirical dependence [9]:

$$
Z_{p}=\frac{m_{o} N^{2} e^{-0,01\left(T-T_{o}\right)}}{0,14 m_{o}(31,416)^{n-1}+\frac{1,87}{z_{n} K_{\varepsilon}}},
$$

where $K_{\varepsilon}$ - the coefficient of geometric shape of the head,

$m_{o}$-the viscosity constant,

$T_{0}$ - the temperature measuring of viscosity constant,

$n$ - the melt yield index.

After the substitution (8) in (7) we get the inequality:

$$
\frac{m_{o} N^{2} e^{-0,01\left(T-T_{o}\right)}}{0,14 m_{o}(31,416)^{n-1}+\frac{1,87}{z_{n} K_{\varepsilon}}} \leq Z_{e n g} C E_{e} .
$$

We transform the inequality (9), leaving the variable $\mathrm{N}$ on the left side: 


$$
N^{2} \leq \frac{Z_{\text {eng }} C E_{e}\left(0,14 m_{o}(31,416)^{n-1}+\frac{1,87}{z_{n} K_{\varepsilon}}\right)}{m_{o} e^{-0,01\left(T-T_{o}\right)}} .
$$

C. Constraint of maximum motor torque for the extruder trouble-free operation:

$$
M_{e} \geq M_{p}
$$

where $\mathrm{M}_{e}$ - the maximum motor torque on the auger shaft of the extruder;

$\mathrm{M}_{P}$ - the working torque of the extruder.

The expression for identifying the working torque on the auger shaft of the extruder can be obtained by reducing the dependence on extrusion power calculation (8) to the torque moment and rotation frequency $Z_{p}=M_{p} \frac{30 \cdot N}{\pi}[10]$ :

$$
M_{p}=\frac{\pi m_{o} N e^{-0,01\left(T-T_{o}\right)}}{30\left(0,14 m_{o}(31,416)^{n-1}+\frac{1,87}{z_{n} K_{\varepsilon}}\right)} .
$$

After identifying the dependence for $M_{p}$, we obtained the following ineaquality:

$$
M_{e} \geq \frac{\pi m_{o} N e^{-0,01\left(T-T_{o}\right)}}{30\left(0,14 m_{o}(31,416)^{n-1}+\frac{1,87}{z_{n} K_{\varepsilon}}\right)} .
$$

We transform the inequality (13), leaving the variable $\mathrm{N}$ on the left side:

$$
N \leq \frac{30 M_{e}\left(0,14 m_{o}(31,416)^{n-1}+\frac{1,87}{z_{n} K_{\varepsilon}}\right)}{\pi m_{o} e^{-0,01\left(T-T_{o}\right)}} .
$$

D. Constraint on the stress rate of the shift between layers.

To guarantee the quality of the product it is necessary to meet the conditions:

$$
[\tau] \geq \tau
$$

where $[\tau]-$ critical stress of the shift $[\tau]=\frac{2 \cdot Q}{N \cdot z_{n}}[11]$

$\tau-$ the stress of the shift on the polymer pipe surface.

The stress of the shift on the polymer pipe surface is defined by the following dependence [12]:

$$
\tau=Q^{n}\left[\frac{2\left(\frac{1}{n}+2\right)}{\pi(R+r)(R-r)^{2}}\right]^{n} 2 m_{o} e^{-0,01\left(T-T_{o}\right)},
$$

where $R$ - the outer radius of the output cross-section;

$r$ - the internal radius of the output cross-section.

After the identification of the dependence for $\tau$ and the substitution together with the temperature processing into the inequality (15) we obtained: 


$$
Q^{1-n} N^{-1} \geq\left[\frac{2\left(\frac{1}{n}+2\right)}{\pi(R+r)(R-r)^{2}}\right]^{n} z_{n} m_{o} e^{-0,01\left(T-T_{o}\right)} .
$$

E. Constraint on the product cooling time.

To guarantee the molding process continuity the correspondence between the cooling time of the product $\tau 4$ in the blown mould, the time of getting the polymer-concrete preform $\tau 1$ and the amount of moulds $p$ are needed:

$$
\frac{\tau 4}{p} \leq \tau 1
$$
[13]:

The dynamics of the product cooling process is described by the following expression

$$
\frac{T_{2}-T}{T_{1}-T}=\left(1-\frac{x}{\sqrt{24 \alpha \tau_{4}}}\right)^{3},
$$

where $T$ - the temperature of the preform;

$T_{1}$ - the temperature of the cooling mould wall;

$T_{2}$ - the final cooling temperature;

$x$ - the thickness of the product;

$\alpha$ - the heat conductivity coefficient;

After the transformation of the given expression into the dependence for defining $\tau 4$ we obtain:

$$
\tau_{4}=\left[\frac{x}{1-\sqrt[3]{\frac{T_{2}-T}{T_{1}-T}}}\right]^{2} / 24 \alpha .
$$

The time of polymer stocking getting $\tau 1$ can be obtained according to the following dependence:

$$
\tau_{1}=\frac{S_{\text {s.c. }} \cdot L}{Q}
$$

where $S_{\text {s.c. }}$ - the area of the output cross-section of the head;

$L-$ the required length of the polymer pipe.

After defining the dependences for $\tau 4, \tau 1$ and specifying the processing temperatures we got the inequality:

$$
Q \leq 24 p \cdot \alpha \cdot S_{6 . c .} \cdot L \cdot\left(\left(1-\sqrt[3]{\frac{T_{2}-T}{T_{1}-T}}\right) / x\right)^{2} .
$$

F. Minimum melt viscosity constraint.

To guarantee the stability and exclusion of the polymer stocking sagging on the output of the coextruder head, it is necessary to introduce the constraint on the minimum melt viscosity of the polymer:

$$
\mu \geq[\mu]
$$

where $\mu$ - the melt viscosity;

$[\mu]$ - the permissible minimum melt viscosity. 
Permissible minimum melt viscosity is defined according to the following formula [14]:

$$
[\mu]=\frac{1,3 \cdot L_{3}^{2} \cdot \rho}{v},
$$

where $\rho$ - the density of melt viscosity,

$\mathrm{v}$ - the linear speed of the preform movement.

The value of the linear speed can be defined on the basis of the volumetric capacity:

$$
v=\frac{Q}{S_{\text {s.c. }}} \text {. }
$$

Thus, the permissible minimum melt viscosity is defined according to the following formula:

$$
[\mu]=\frac{1,3 \cdot L_{3}^{2} \cdot \rho \cdot S_{\text {s.c. }} .}{Q} .
$$

The viscosity of the polymer melt is identified by the following formula:

$$
\mu=m_{o} e^{-0,01\left(T-T_{o}\right)} .
$$

Having defined the dependences for $\mu,[\mu]$ we get the following inequality:

$$
Q \geq \frac{1,3 \cdot L_{3}^{2} \cdot \rho \cdot S_{6 . c .}}{m_{o} e^{-0,01\left(T-T_{o}\right)}}
$$

The constraints (13) and (17) are nonlinear. To do the optimization task of the combined extrusion of the blown containers with the usage of the blown rotor by the method of nonlinear programming [15], all the constraints are brought to the form:

$$
\left.\begin{array}{l}
A_{1} X+C_{1} Y \leq B_{1} \\
A_{n} X+C_{n} Y \leq B_{n}
\end{array}\right\} .
$$

For this purpose we find the logarithms (10) and (12):

$$
\left.\begin{array}{l}
X_{1}=\lg M_{p} \\
X_{2}=\lg Q
\end{array}\right\} .
$$

We also find the logarithm for the linearization of the objective function:

$$
K_{o}=\lg Q=X_{1} \Rightarrow \max
$$

Thus, we have elaborated the mathematical model and the system of constraints of coextrusion molding, in which the target function is presented as the optimality criterion of the technological process that allows defining the optimum process-dependent parameters of combined processing of the primary, secondary polymer raw material and concrete. As a result, the task of improving the process of coextrusion molding of the products is brought to linear programming.

The usage of the coextrusion machine can by presented by two main trends:

- the immediate optimization of process-dependent parameters: preform molding pressure, the auger rotation frequency and the efficiency according to the set-up parameters of the equipment construction and processing conditions;

- obtaining the dependences of the influence of different design factors and processdependent parameters on the efficiency and quality of the manufactured products.

Thus, due to the study of the technological process of the coextrusion molding of the given mathematical model we can fully optimize the whole process of manufacturing new products. 


\section{Discussion}

One of the important processes going on in the process of coextrusion molding is the preform cooling [16]. Its cooling time is one of the technological cycle components, and consequently it influences the maximum efficiency. We research the dynamics of the preform cooling process in the mould by mathematical modeling. The main arguments of the process are the volume of the preform, the type of raw material, and the preform thickness. The results of the mathematical modelling are presented by Fig. 1.

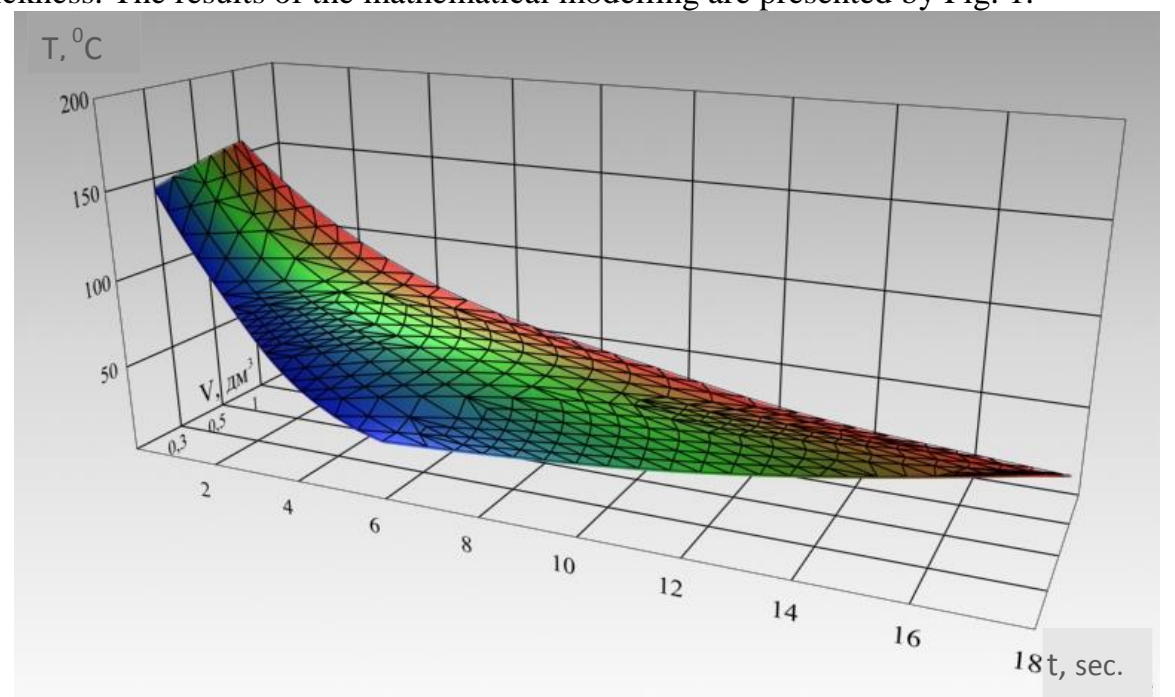

Fig. 1. Dynamics of cooling process of the preform in the mould of the coexstrusion machine: $\mathrm{V}\left(\mathrm{dm}^{3}\right)$ - the volume of the product; $\mathrm{t}$ - the time of cooling of the preform in the mould, $\mathrm{T}-$ the preform temperature.

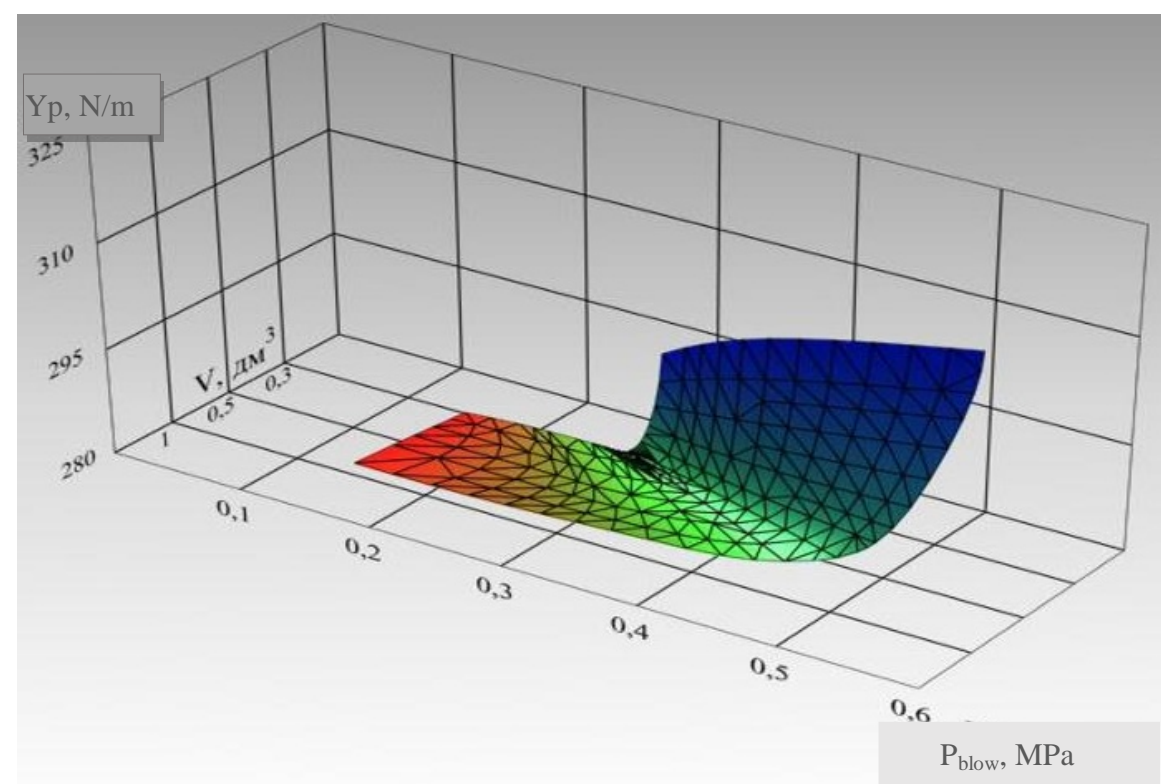

Fig. 2. The study of the blow pressure influence on the plybond strength in the multilayer polymer system while manufacturing different volume products: $\mathrm{Y}_{\mathrm{p}}$ - the plybond strength; $\mathrm{P}_{\text {blow }}$ - the blow pressure; $\mathrm{V}$ - the product volume $\left(\mathrm{dm}^{3}\right)$. 
Figure 2 presents the research of layers fusion forming process in the multilayer system. It is shown that the layers fusion forming in the given system occurs according to the microreaction mechanism [17]. The temperature, the blow pressure and the contact continuation during the structure forming influence greatly the intensity of microreaction processes behaviour [18-20]. The linear dependence of the joint efficiency on the blow pressure is exposed. That is why the relevant optimization of the process-dependent parameters was done to provide the necessary level of the layers joint.

The joint efficiency is estimated by the plybond strength $\mathrm{Y}_{\mathrm{p}}$. The level of layers interaction in the polymer-concrete system determines the operating characteristics of the multilayer material considerably.

The recommended process-dependent parameters of layers joint for the coextrusion High Density Polyethylene - High Density Polyethylene - Lime are as follows:

1. The technological process has to be carried out with the maximum possible, close to the critical, stress of the shift in the multilayer flow. The maximum possible total efficiency, when the critical value of shift deformations is achieved, is:

- -for the gap of $1 \mathrm{~mm}$ with the internal channel radius of $8 \mathrm{~mm}$ and the temperature $170{ }^{0} \mathrm{C}-4 \mathrm{~g} / \mathrm{s}$, the maximum stress of the shift $-312 \mathrm{MPa}$, the speed of the shift $538 \mathrm{c}^{-1}$;

- for the gap of $4 \mathrm{~mm}$ with the internal channel radius of $8 \mathrm{~mm}$ and the temperature 170

${ }^{0} \mathrm{C}-7 \mathrm{~g} / \mathrm{s}$, the maximum stress of the shift $-364 \mathrm{MPa}$, the speed of the shift $575 \mathrm{c}^{-1}$.

2. The pressure of blow molding has to be maintained at $0,25-0,35 \mathrm{MPa}$, in this case the minimum initial speed of deforming will be guaranteed, and at the same time this pressure will be enough for pressing the polymer container to the walls of the mould, which is necessary for the quick cooling of the preform.

\section{Conclusions}

1. The study estimated the limits and optimization criterion for operating the coextrusion machine for forming polymer-concrete building constructions with the purpose of defining the metrics and output parameters of the mathematical model on the basis of the record of the auger rotation frequency, the maximum motor torque, the stress of the shift, the product time cooling, and the minimum melt viscosity constraint.

2. We elaborated the algorithm and the mathematical model of coextrusion molding process with the usage of singled out constraints and the target function with the purpose of design factors and process-dependent parameters of the coextrusion machine optimization.

3. According to the result of the deflected mode, the optimal value of the blow pressure of $0,5 \mathrm{MPa}$, providing the even layup, is defined.

The study was carried out with the financial support of the Ministry of Education and Science of the Russian Federation within the framework of the scientific project 10.1622.2017/4.6.

\section{References}

1. V. Dyadichev, A. Kolesnikov, A. Dyadichev, S. Menyuk, E. Dyadicheva, MATEC Web Conf., VI International Scientific Conference IPICSE-2018, 251 (2018)

2. V.V. Dyadichev, A.V. Kolesnikov, S.G. Menyuk, A.V. Dyadichev, IOP Conference Series “Applied Mechanics and Systems Dynamics” (Omsk, 2018) (to be published)

3. N.V. Lyubomirskiy, S.I. Fedorkin, A.S. Bakhtin, T.A. Bakhtina, MCRJ, 23, 3 (2017)

4. S. Fic, N.V. Lyubomirskiy, D. Barnat-Hunek, Materials Science Forum: "Materials and Technologies in Construction and Architecture”, 931 (2018)

5. A. Gusev, V. Shul'gin, E. Braga, E. Zamnius, G. Starova, K. Lyssenko, I. Eremenko, W. Linert, Journal of Luminescence, 202 (2018) 
6. A.N. Gusev, V. F. Shul'gin, E.V. Braga, I. Nemec, B.F. Minaev, G.V. Baryshnikov, Z. Trávníček, H. Ågren, I. L. Eremenko, K. A. Lyssenko, W. Linert, Polyhedron (2018)

7. V. Shul'gin, N. Pevzner, A. Gusev, M. Sokolov, V. Panyushkin, J. Devterova, K. Kirillov, I. Martynenko, W. Linert, Journal of Coordination Chemistry (2018).

8. V.S. Yankovskaya, I.I. Dovhyi, N.A. Bezhin, V.V. Milyutin, N.A. Nekrasova, S.V. Kapranov, V.F. Shulgin, Journal of Radioanalytical and Nuclear Chemistry, 318, 2 (2018)

9. V.V. Dyadichev, A.V. Kolesnikov, A.V. Dyadichev, E.A. Dyadicheva, Construction and Technogenic Safety: Scientific and Technical Journal of Construction and Architecture, 10, (62) (2018)

10. E.A. Malitskova, I.I. Potapov, Plastics Waste Recycling (Moscow, Avis Original, 1997)

11. J. Fogarty, C.J. Rauwendaal, D. Fogarty, A. Rios, SPE ANTEC Techn. Papers (2001)

12. C. Rauwendaal, Polymer extrusion (Munich, Hauser Garduer, 2001)

13. J. Pape, H. Potente, C. Obermann, 15th Annual Meeting of the Polymer Processing Society (Den Bosch, the Netherlands, 1999)

14. B. Davis et al., Polym. Eng. Sci., 38, 7 (1998)

15. C.D. Han Multiphase flow in polymer processing (New York: Academic Press, 1981)

16. P. Elemans, 58th SPE ANTEC (2000)

17. P. Elemans, J.M. van Wunnik, 58th SPE ANTEC (2000)

18. R.T. Steller, Polym. Eng. Sci., 30, 7 (1990)

19.V.S. Kim, Dispersing and mixing in plastics production processes (Moscow: Chemistry, 1988)

20. P.A. Shvetsov, Technology of processing plastics (Moscow: Chemistry, 1988) 